\title{
The prevalence of abnormal findings in screening CT brains performed on patients admitted with psychiatric symptoms
}

Authors:
Praniel Bennimahadeo
Jaynund Maharajh ${ }^{1}$
Affiliations:
'Department of Radiology,
King Edward VIII Hospital,
Nelson R Mandela School of
Medicine, University of
KwaZulu-Natal, South Africa
Corresponding author:
Praniel Bennimahadeo,
drpbennimahadeo@
webmail.co.za
Dates:
Received: 10 Jan. 2016
Accepted: 20 Sept. 2016
Published: 22 Nov. 2016
code with your
How to cite this article:
mobile device
to read online.
Bennimahadeo P, Maharajh J.
The prevalence of abnormal
findings in screening CT
brains performed on patients
admitted with psychiatric
symptoms. S Afr J Rad.
2016;20(1), a976. http://
dx.doi.org/10.4102/sajr.
v20i1.976
Copyright:
C 2016. The Authors.
Licensee: AOSIS. This work
is licensed under the
Creative Commons
Attribution License.

Background: No clear guidelines exist regarding the role of computerised tomography $(\mathrm{CT})$ as a screening neuroimaging tool in psychiatric practice. The aim of this study was to evaluate the usefulness of historical and clinical parameters on the CT request form, identify the spectrum of CT findings and correlate these to develop guidelines for the use of screening CT brains in patients who present with psychiatric symptoms.

Methods: Requests and reports for the brain CT's of 507 consecutive patients over a 2-year period, between 2013 and 2014, referred from a psychiatric institute for screening CT brain scans, were reviewed. Analysis was performed for the history of trauma and seizures, Glasgow Coma Scale (GCS) score, focal neurological signs, papilloedema, electroencephalograph, relevant blood results and abnormal CT findings. All reports were approved by a consultant radiologist.

Results: No abnormality was noted in $69 \%$ of CT scans. Cerebral atrophy, infarcts, cysts and calcific foci were present in $30 \%$ of patients. One patient presenting with focal neurology had a CT demonstrating an extradural haematoma which required neurosurgical intervention. No focal brain lesions, potentially responsible for the psychosis, were identified in any other patient.

Conclusion: Routine CT screening of patients who present with psychotic symptoms, in the absence of focal neurological deficit, does not add value to patient outcome, but rather contributes to the escalating health care expenses and unnecessary radiation dose. CT screening of psychiatric patients should be reserved for patients with reliable predictors of intracranial abnormalities such as lateralising signs, seizures, persistent or worsening headaches; decrease in GCS, papilloedema and in patients where the onset of symptoms occurred at an age above 50 .

\section{Introduction}

According to The South African Federation of Mental Health, approximately one in five South Africans suffer from a mental illness, and approximately $25 \%$ of all general practitioner consultations are because of psychiatric conditions. ${ }^{1}$ Many of these patients will be referred to a psychiatrist or undergo a battery of investigations to exclude an organic aetiology for their presenting symptoms. Computerised tomography (CT) is used extensively in psychiatric practice as a neuroimaging screening tool to identify possible intracranial pathology. The average cost of a CT brain scan is between R3000 and R5000 in South Africa. The approximate effective radiation dose of a contrasted CT brain scan is 4 millisieverts (mSv), which is comparable to 200 chest X-rays $(0.02 \mathrm{mSv})$. These estimates highlight the potential burden of disease associated with psychiatric conditions. There is also the increased radiation exposure and its risk to patients must be weighed against the possible benefit of investigative CT brain scans in the psychiatric patient population.

There are no official South African guidelines regarding the use of CT as an investigative tool in psychiatric patients. Much debate has occurred with regard to appropriate screening tests in patients who present with psychiatric symptoms. The Royal Australian and New Zealand College of Psychiatrists (RANZCP) recommend routine neuroimaging for patients presenting with firstepisode psychosis. They do, however, acknowledge that evidence regarding the possibility of routine CT imaging altering patient outcomes or clinical management is currently not available and needs to be explored. ${ }^{2}$ 


\section{Aim}

The study aimed to determine the spectrum of abnormalities seen on brain CT scans of psychiatric patients for whom CT screening was performed. The study was undertaken in order to provide preliminary data for the development of guidelines for the use of CT in this cohort. The objectives of the study were to:

- identify the demographic, historical and clinical information,

- assess the indications on the CT request form, and

- describe the spectrum of findings of the CT brain screenings.

\section{Method}

Townhill Hospital is a provincial facility specialising in mental health care located in Pietermaritzburg, KwaZuluNatal Province. The CT brain scans that are requested by Townhill's psychiatrists are performed at the neighbouring Grey's Hospital, which is a tertiary level referral and teaching institution. The electronic request forms and reports for inpatients referred for psychiatric screening CT brain scans from Townhill Hospital were retrospectively analysed for 507 consecutive CT brain scans performed over a 2-year period, between 2013 and 2014. This information was retrieved from the computerised appointment records and the radiology information system that links scanned hand-written request forms to the radiology report and $\mathrm{CT}$ images. The forms were reviewed for the presence or absence of the following information: demographic (age and gender), historical (history of trauma, seizures), clinical examination (Glasgow Coma Scale [GCS] score, focal neurological signs, papilloedema), clinical investigations (electroencephalograph results and relevant blood tests as shown below) and indication for the study. The abnormal CT scan report findings were also captured, especially those that could cause psychosis and alter the patient's management. All reports were performed by trained staff and approved by a consultant radiologist. Approval was obtained from the University of KwaZulu-Natal Biomedical Research Ethics Committee (Ref: BEO19/14) and the hospital. Patient informed consent was not required.

Numeric data were summarized using medians and interquartile range (IQR) or means and standard deviations as appropriate, categorical data by frequencies and percentages. Two group comparisons of categorical data were made using chi-squared test or Fisher's exact test as appropriate.
A significance level of $p<0.05$ was used for statistical testing of significance. The analyses were performed utilising the Stata v 13.1, StataCorp College Station.

\section{Results}

Of the 507 records reviewed, 279 male (median age 29 years) and 228 female patients (median age 35.5 years) were evaluated. The highest number of men were in the 25-34 age group (33\%) and the highest number of women were in the above 45 age group (34\%).

The age distribution of women was significantly different to the men $(p=0.001)$, and the median age of women (35.5) was significantly greater than that of men (29). A contributing factor may have been due to the increased substance use in the younger male cohort (Table 1).

The clinical history and motivation for the investigation were absent in the vast majority of patients referred. Relevant information such as a history of trauma was not provided in 448 patients $(88 \%)$. No information about previous seizures was provided in 500 patients $(98 \%)$. Clinical information relating to the GCS score, focal neurological signs and papilloedema and results of an electroencephalograph and blood tests were not provided in more than $93 \%$ of patients (Table 2).

There were various indications for requesting the CT scans; $75 \%$ were requested to exclude a general medical condition (GMC) with no supporting laboratory or clinical information. Other patients had multiple indications, for example, to exclude a GMC and to exclude a substance-induced psychosis. Twelve patients did not have any indication provided (Table 3 ).

Taking into account that patients had multiple indications, the most common reasons for requesting the $\mathrm{CT}$ scan were to exclude GMCs or organic causes in 336 patients, first onset of psychosis in 190 patients, known psychosis in 161 patients and mood disorders in 138 patients as shown in Figure 1.

TABLE 1: Age and gender distribution.

\begin{tabular}{lccc}
\hline Age category & Male & Female & Total \\
\hline$<25$ years & 89 & 56 & 145 \\
$25-34$ years & 92 & 51 & 143 \\
$35-44$ years & 44 & 44 & 88 \\
+45 years & 54 & 77 & 131 \\
\hline Total & 279 & 228 & 507 \\
\hline
\end{tabular}

TABLE 2: Relevant history and clinical data provided on the CT request form.

\begin{tabular}{|c|c|c|c|c|c|c|c|}
\hline Clinical data & & Positive & Negative & No data provided & Abnormal & Normal & No data provided \\
\hline \multirow[t]{2}{*}{ Relevant history } & Previous trauma & 52 & 7 & 448 & - & - & - \\
\hline & Seizures & 7 & 0 & 500 & - & - & - \\
\hline \multirow{3}{*}{$\begin{array}{l}\text { Clinical examination } \\
\text { findings }\end{array}$} & GCS $15 / 15$ & 2 & 0 & 505 & - & - & - \\
\hline & Focal signs & 1 & 28 & 478 & - & - & - \\
\hline & Papilloedema & 0 & 0 & 507 & - & - & - \\
\hline \multirow[t]{2}{*}{ Clinical investigations } & EEG & - & - & - & 1 & 5 & 501 \\
\hline & Blood & - & - & - & 2 & 30 & 475 \\
\hline
\end{tabular}

CT, computerised tomography; GCS, Glasgow Coma Scale, EEG, electroencephalogram. 
Of the 507 cases, 348 (69\%) had no intracranial abnormality noted on the CT scan. Of these 348 patients, four had extracranial abnormalities including a scalp foreign body, sinusitis, a previous burr hole defect and an old depressed skull fracture. In the studies demonstrating pathology, 111 cases (21\%) demonstrated cerebral atrophy, 18 cases $(4 \%)$ had calcific foci, 24 patients $(5 \%)$ had infarcts and 6 patients $(1 \%)$ were found to have cysts. Many patients with cerebral atrophy do not have psychosis, and even though there is an association between schizophrenia and global atrophy, ${ }^{3}$ this was not stated as an indication for referral on the request form. The one patient with a positive focal sign had a proven parietal extradural haematoma (Figure 2).

\section{Discussion}

In this study, CT has been implemented as a routine investigation for a significant majority of psychiatric patients to exclude possible organic pathology. In this situation, radiologists rely on the clinical information provided by clinicians to advise on the use and timing of appropriate investigations. The patients involved in this study were appointed CT scan dates via an elective booking system. The lack of relevant trauma and seizure history, as well as of significant clinical and laboratory investigations provided, prevented the appropriate selection of patients who may have benefitted from early utilisation of CT scans of the brain to identify a treatable aetiology for their symptoms. It further increased the duration of the waiting time for patients to be

\begin{tabular}{lcc}
\multicolumn{2}{l}{ TABLE 3: The number of indicators per patient. } \\
\hline $\begin{array}{l}\text { Number of indicators per } \\
\text { patient }\end{array}$ & Number of patients & $\%$ \\
\hline 0 & 12 & 2 \\
1 & 380 & 75 \\
2 & 105 & 21 \\
3 & 10 & 2 \\
\hline
\end{tabular}

scanned and added to the workload of the scanner, all escalating the burden on our strained health system.

The majority of CT scans performed in this study were normal, indicating a discordance between psychosis and abnormal findings on CT scans. However, there are prior studies that refute this. In the 2007 South African study by Jeenah and Moosa, ${ }^{3}$ conducting CT screening was encouraged as a central nervous system disorder may initially manifest as a psychiatric symptom with a treatable cause such as an intracranial tumour, which can be diagnosed by CT and may otherwise be missed. They studied 55 eligible CT scans of 600 psychiatric patients and concluded that abnormalities were found in $20(36.4 \%)$. Of these abnormalities, six had old infarcts, seven had global atrophy, one had sustained a trauma and six were grouped with the following conditions: pituitary adenoma, tuberculous granuloma and neurocysticercosis. This study differed from Jeenah's study, evaluating 507 CT scans compared to 55 CT scans. The incidence of cerebral atrophy was $21 \%$ in this study compared to $12 \%$ in their study.

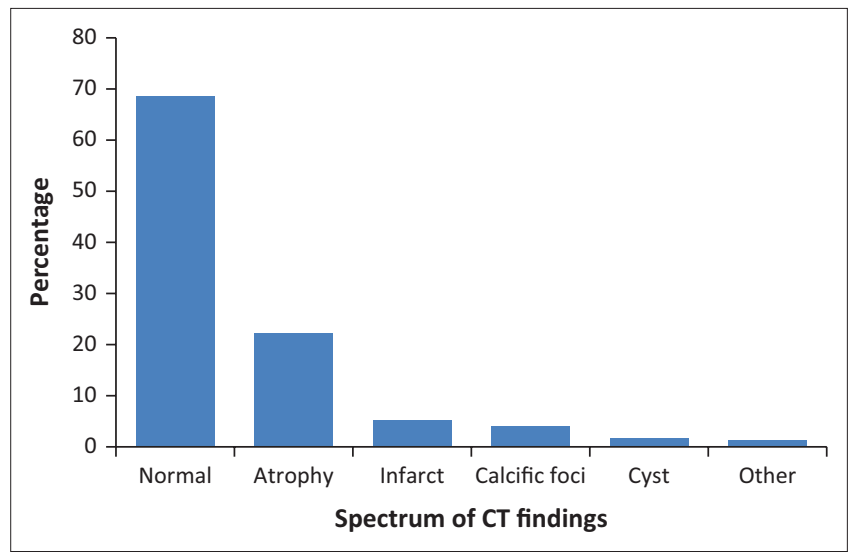

FIGURE 2: Spectrum of computerised tomography results.

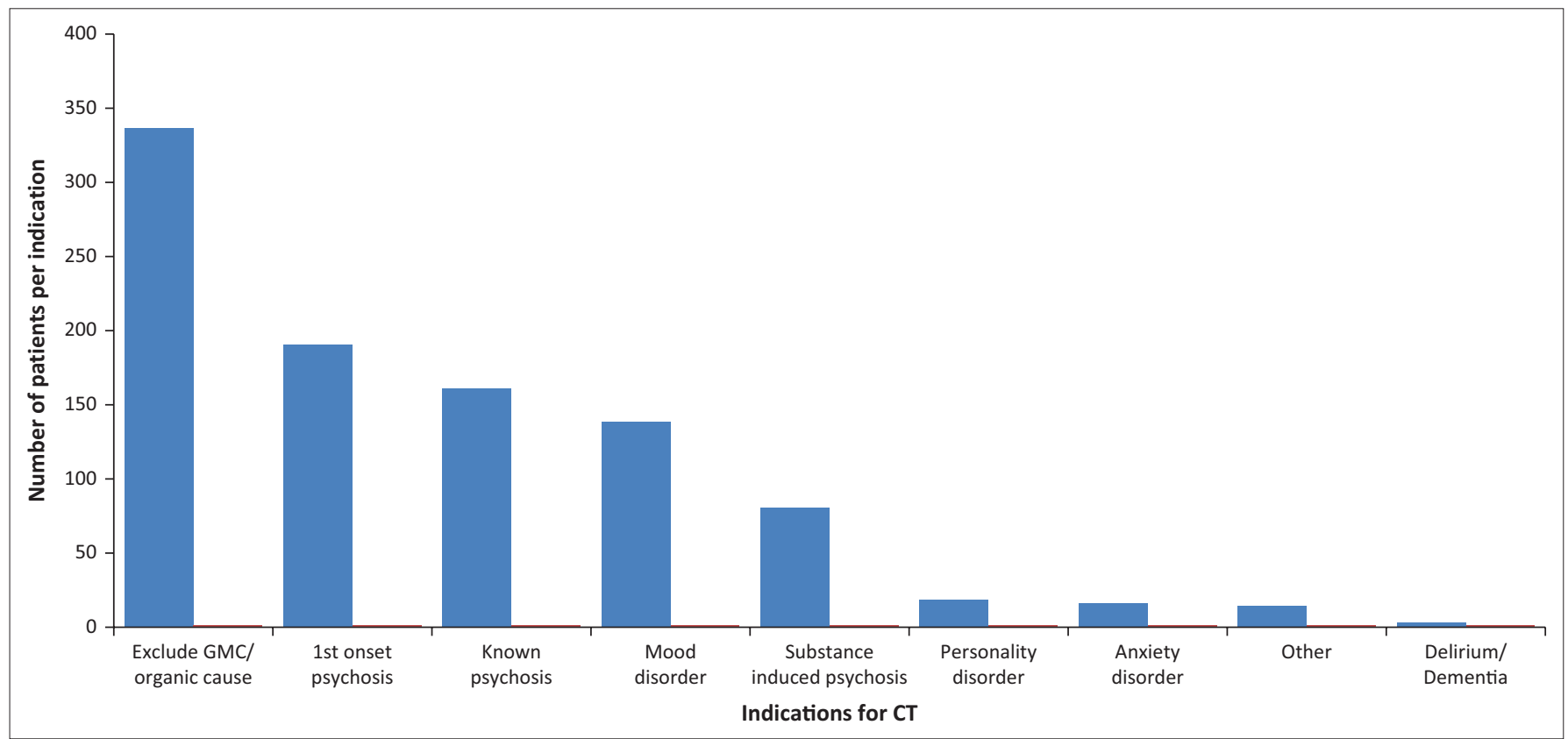

FIGURE 1: Indications for requesting computerised tomography brain scans. 
Similarly, an article by Andreasen from the Department of Psychiatry at the University of Iowa, College of Medicine, stated that ventricular enlargement and cortical atrophy are abnormalities noted on CT that assist in diagnosing schizophrenia and implicate a structural cause of the disease, thus supporting the need to conduct CT screening of psychiatric patients. However, managing schizophrenic patients with or without ventricular enlargement or cortical atrophy remains the same. ${ }^{4}$

McClellan et al. ${ }^{5}$ conducted a comprehensive retrospective audit of the CT brain scans of 261 patients admitted with psychiatric symptoms over a 3-year period, of whom $88 \%$ were normal and $12 \%$ were found to be abnormal because of cortical atrophy, basal ganglia calcifications and old lacunar infarction, all of which were unrelated to the patient's psychiatric condition. Their results showed that there was no justification for routinely conducting CT scans for psychiatric inpatients in the absence of focal neurological signs. In concordance with the above findings was Khandapour et al., ${ }^{6}$ who reviewed 112 consecutive MRI brain scans and 204 consecutive CT brain scans performed on patients with first onset psychosis, the aim being to determine whether imaging was associated with early detection of organic causes of psychosis. Their conclusion revealed that routine magnetic resonance imaging (MRI) and CT brain scans performed on patients without focal neurology were unlikely to identify a disease process and did not impact on their management.

The performance of the published criteria used by psychiatrists, $^{7}$ whereby CT brain scans were used in all patients presenting with schizophrenia or first onset psychosis, was critiqued by Rock and Owen. ${ }^{8}$ Their study consisted of a 3-year retrospective case note audit of 143 patients (one abnormality was noted that did not alter the diagnosis or management of the patient), which revealed that the efficacy of the published criteria was not supported. They recommended that new clinically relevant guidelines for the use of CT scans be developed.

Supporting the above findings was a study by Agzarian et al., 9 who retrospectively assessed 397 consecutive CT brain scans of patients who presented with psychiatric symptoms without focal neurology over a 2-year period. No abnormalities were found in $95 \%$ of the scans, with 5\% showing non-specific minor abnormalities that were followed up by MRI, which showed no relevant abnormality, whereas those noted on the CT scan were considered to be unrelated to the patient's psychiatric condition. In their conclusion, Agzarian et al. made mention of two important factors, the cost of performing the 397 scans (approximately 6000 Australian Dollars), with little apparent benefit related to the patient's management, and the risk of radiation exposure outweighing any real assistance to their management.

Similarly, Strahl et al. ${ }^{10}$ identified inconsistencies in the worldwide recommendation of brain CT algorithms of 237 patients presenting with first onset psychotic symptoms without focal neurological signs who had CT scans. All reports were approved by a consultant radiologist, and the results did not identify any focal brain lesion potentially responsible for the psychosis or focal lesion requiring surgical intervention in any patient. Hrabrić et al., ${ }^{11}$ Goulet et al. ${ }^{12}$ and Bain ${ }^{13}$ reached related conclusions.

Psychiatric geriatric populations ranging between 55 and 90 years were evaluated by Rozenbilds and Gilchris ${ }^{14}$ who had similar findings to Hollister and Harris ${ }^{15}$ in demonstrating that factors which correlated strongly with an abnormal CT were a recent alteration in behaviour or personality or a first psychotic event after the age of 50 .

The current study also found CT scans to be of little benefit, with clinical examination, history of seizures, focal neurological deficit and age at onset of symptoms being more specific in predicting positive causative outcomes, thus supporting the role of CT in refuting or confirming abnormal findings of other clinical investigations. The low rate of organic abnormalities, high cost low yield and exposure to radiation warrant consideration of the value of $\mathrm{CT}$ as an investigative tool (Table 4).

\section{Proposed guidelines for workup of a psychiatric patient}

This study has revealed similar findings to the authors McClellan et al., ${ }^{5}$ Khandapour et al., ${ }^{6}$ Rock and Owen, ${ }^{8}$ Agzarian et al., ${ }^{9}$ Strahl et al., ${ }^{10}$ Hrabrić et al., ${ }^{11}$ Goulet et al. ${ }^{12}$

TABLE 4: CT findings in previous psychiatric population studies.

\begin{tabular}{|c|c|c|c|c|c|c|c|}
\hline \multirow[t]{3}{*}{ Study } & \multirow[t]{3}{*}{ Year } & \multirow{3}{*}{$\begin{array}{l}\text { Total no. of } \\
\text { patients }\end{array}$} & \multicolumn{4}{|c|}{ CT brain findings } & \multirow[t]{3}{*}{ Spectrum of abnormalities or comment } \\
\hline & & & \multicolumn{2}{|c|}{ Normal } & \multicolumn{2}{|c|}{ Abnormal } & \\
\hline & & & $n$ & $\%$ & $n$ & $\%$ & \\
\hline Khandapour et al. ${ }^{6}$ & 2013 & 204 & 68 & 33.3 & 136 & 66.7 & Atrophy, infarcts and cysts. \\
\hline Strahl et al. ${ }^{10}$ & 2010 & 237 & 192 & 82.4 & 45 & 17.6 & $\begin{array}{l}\text { Small vessel ischaemic changes, arachnoid cyst, cerebral atrophy. No } \\
\text { alteration in clinical management. }\end{array}$ \\
\hline Jeenah and Moosa ${ }^{3}$ & 2007 & 55 & 35 & 64.0 & 20 & 36.4 & $\begin{array}{l}6 \text { infarcts; } 7 \text { atrophy; } 1 \text { previous trauma; } 6 \text { grouped with } \\
\text { neurocysticercosis, TB granuloma and pituitary adenoma. }\end{array}$ \\
\hline Goulet et al. ${ }^{12}$ & 2006 & 384 & - & - & - & 1.3 & In first-episode psychosis, routine CT or MRI scans are of little benefit. \\
\hline Agzarian et al. ${ }^{9}$ & 2006 & 397 & 377 & 95.0 & 20 & 5.0 & $\begin{array}{l}\text { Abnormalities were found to be clinically unrelated to the patient's } \\
\text { condition. }\end{array}$ \\
\hline Rock and Owen ${ }^{8}$ & 2003 & 143 & 120 & 83.9 & 1 & 0.6 & No changes to diagnosis or management. \\
\hline Bain $^{13}$ & 1998 & 127 & 123 & 96.0 & 4 & 4.0 & $\begin{array}{l}\text { The abnormal cases had MRI scans that showed no abnormality. CT } \\
\text { results did not alter management or outcome. }\end{array}$ \\
\hline McClellan et al. ${ }^{5}$ & 1988 & 261 & 229 & 88.0 & 32 & 12.0 & Atrophy and infarcts. \\
\hline
\end{tabular}

$\mathrm{CT}$, computerised tomography; TB, tuberculous; MRI, magnetic resonance imaging. 
and Bain, ${ }^{13}$ who are against using CT as a screening neuroimaging tool. Relevant laboratory investigations are more cost effective than advanced radiological tests and could be used instead of conducting a CT scan. CT scans are not only expensive but also expose the patient to radiation. This study therefore recommends using patient demographics, that is, age at presentation, GCS score, focal neurological signs, seizures and papilloedema, as clinical criteria to warrant the use of CT. Guidelines have been proposed in the workup of a psychiatric patient noting that $\mathrm{CT}$ brain is not routinely indicated with an emphasis on history, physical examination and relevant laboratory investigations to inform such a decision. ${ }^{16}$

In this study, one patient with a focal neurological sign required intervention and an organic cause was identified. It has been recommended that a patient presenting with the following history, symptoms and signs warrants a CT brain:

- age at onset of symptoms greater than 50 years ${ }^{14,15}$

- history of head trauma or seizures ${ }^{5}$

- persistent or increasing headache ${ }^{14}$

- decrease in the GCS

- presence of focal neurological signs ${ }^{5}$

- presence of papilloedema $^{5}$

\section{Conclusion}

The limitations identified in this study are that patients were limited to referrals from a single hospital, this is a retrospective study of referred patients and case ascertainment was not controlled. The evidence from this study indicates that routine requests for $\mathrm{CT}$ brain scanning of patients who present with psychotic symptoms in the absence of focal neurological deficit do not add value to patient outcome, but rather contribute to the escalating health care expenses and increase their radiation dose. CT of the brain should be carefully considered within the context of a clinical presentation of psychosis.

\section{Acknowledgements}

The authors thank Dr D. Naidoo, Department of Anaesthetics, University of KwaZulu-Natal for help with data collection and analysis and the staff of Greys Hospital for their assistance.

\section{Competing interests}

The authors declare that they have no financial or personal relationships which may have inappropriately influenced them in writing this article.

\section{Authors' contributions}

P.B. was responsible for conceptualising the project, drafting the research protocol and journal article and completing the data collection. J.M. supervised the study, made conceptual contributions, facilitated interpretation of the results and edited the research protocol and final manuscript.

\section{References}

1. Xoliswa Zulu, Mental health on the rise in South Africa. Health Systems Trust website http://www.hst.org.za/news/mental-illnesses-rise-sa

2. Royal Australian and New Zealand College of Psychiatrists Clinical Practice Guidelines Team for the Treatment of Schizophrenia and Related Disorders. Royal Australian and New Zealand College of Psychiatrists clinical practice guidelines for the treatment of schizophrenia and related disorders. Aust N Z J Psychiatry. 2005;39:1-30. http://dx.doi.org/10.1111/j.1440-1614.2005.01516.x

3. Jeenah FY, Moosa MYH. CT scans in psychiatric patients- an exploratory study at Chris Hani Baragwanath Hospital. SAJP. 2007;13(1):22-25, 88.

4. Andreasen N. Brain imaging: Applications in psychiatry. Science. 1988;239: 1381-1388.

5. McClellan RL, Eisenberg RL, Giyanani VL. Routine CT screening of psychiatric inpatients. Radiology. 1988;169:99-100. http://dx.doi.org/10.1148/radiology.169.1.3420286

6. Khandapour N, Hoggard N, Connoly DIA. The role of MRI and CT of the brain in first episode of psychosis. Clin Radiol. 2013;68:245-250.

7. Weinberger DR. Brain disease and psychiatric illness: When should a psychiatrist order a CAT scan? Am J Psychiatry. 1984;141(12):1521-1527. http://dx.doi. org/10.1176/ajp.141.12.1521

8. Rock JD, Owen PW. An investigation of criteria used to indicate cranial CT in males with schizophrenia. Acta Neuropsychiatr. 2003;15:284-289. http://dx.doi. with schizophrenia. Acta Neuropsychia
org/10.1034/j.1601-5215.2003.00041.x

9. Agzarian MJ, Chryssidis S, Davies RP, Pozza CH. Use of routine computed tomography brain scanning of psychiatry patients. Australas Radiol. 2006;50: 27-28. http://dx.doi.org/10.1111/j.1440-1673.2005.01542.x

10. Strahl B, Cheung Y, Stuckey S. Diagnostic yield of computed tomography of the brain in first episode psychosis. J Med Imaging Radiat Oncol. 2010;54:431-434.

11. Hrabrić K, Jukić V, Bilić $P$, Celić $I$, Herceg M. Neuroradiologic diagnostics in patients hospitalized in Vrapce Psychiatric Hospital. Gen Hosp Psychiatry. 1998;20(5):282-291.

12. Goulet K, Deschamps B, Evoy F, Trudel JF. Use of brain imaging (computed tomography and magnetic resonance imaging) in first-episode psychosis: Review and retrospective study. Can J Psychiatry. 2009;54(7):493-501.

13. Bain BK. CT scans of first-break psychotic patients in good general health. Psychiatr Serv. 1998;49:234-235. http://dx.doi.org/10.1176/ps.49.2.234

14. Rozenbilds UY, Gilchris PN. The value of CT Head Scan in Elderly Psychiatric Patients. Int J Geriatr Psychiatry. 1989;4:155-157.

15. Hollister LE, Harris NB. Clinical use of CT and MR Scans in Psychiatric Patients. J Psychiatry Neurosci. 1991;16(4):194-198.

16. Assessment of Psychosis. Step by step diagnostic approach [homepage on the Internet]. BMJ [cited 2015 Sept 17]. Available from http://bestpractice.bmj.com/ best-practice/monograph/1066.html 